\title{
Pharmacist-physician collaborative care for outpatients with left ventricular assist devices using a cloud-based home medical management information-sharing system: a case report
}

Yoshiki Katada', Atsushi Yonezawa', Momoe Utsumi², Noriaki Kitada', Yu-ki Sato', Katsuyuki Matsumura', Asami Sukeishi', Shunsaku Nakagawa' ', Satoshi Imai', Takayuki Nakagawa' ${ }^{1}$, Kenji Minakata ${ }^{3}$, Hideo Kanemitsu', Kenji Minatoya ${ }^{3}$, Shinichi Nomoto ${ }^{4}$ and Kazuo Matsubara ${ }^{1 *}$

\begin{abstract}
Background: The standard anticoagulation therapy for patients implanted with left ventricular assist devices (LVADs) includes warfarin therapy. We developed a cloud-based home medical management information-sharing system named as LVAD@home. The LVAD@home system is an application designed to be used on iPad tablet computers. This system enables the sharing of daily information between a patient and care providers in real time. In this study, we reported cases of outpatients with LVADs using this system to manage anticoagulation therapy.

Case presentation: The patient, a man in his 40 s with end-stage heart failure owing to non-ischemic dilated cardiomyopathy, underwent LVAD implantation and warfarin was started on postoperative day 1. He started to use LVAD@home to manage warfarin therapy after discharge (postoperative day 47). He sent his data to care providers daily. By using this system, the pharmacist observed his signs of reduced dietary intake 179 days after discharge, and after consulting the physician, told the patient to change the timing of the next measurement earlier than usual. On the next day, the prothrombin time-international normalized ratio increased from 2.0 to 3.0, and thus the dose was decreased by $0.5 \mathrm{mg}$. Four patients used this system to monitor warfarin therapy from October 2015 to March 2018. In these patients, the time in therapeutic range was $90.1 \pm 1.3$, which was higher than that observed in previous studies. Additionally, there were no thromboembolic events or bleeding events.
\end{abstract}

Conclusions: The cloud-based home management system can be applied to share real-time patient information of factors, including dietary intake that interact with warfarin. It can help to improve long-term anticoagulation outcomes in patients implanted with LVAD.

Keywords: Warfarin, Anticoagulation, Pharmacist, Prothrombin time-international normalized ratio, Self-testing, Left ventricular assist device, CoaguCheck ${ }^{\circledR}$ X, Cloud-based home management system

\footnotetext{
* Correspondence: kmatsuba@kuhp.kyoto-u.ac.jp

'Department of Clinical Pharmacology and Therapeutics, Kyoto University Hospital, 54 Kawahara-cho, Shogoin, Sakyo-ku, Kyoto 606-8507, Japan

Full list of author information is available at the end of the article
}

C C The Author(s). 2021 Open Access This article is licensed under a Creative Commons Attribution 4.0 International License, which permits use, sharing, adaptation, distribution and reproduction in any medium or format, as long as you give appropriate credit to the original author(s) and the source, provide a link to the Creative Commons licence, and indicate if changes were made. The images or other third party material in this article are included in the article's Creative Commons licence, unless indicated otherwise in a credit line to the material. If material is not included in the article's Creative Commons licence and your intended use is not permitted by statutory regulation or exceeds the permitted use, you will need to obtain permission directly from the copyright holder. To view a copy of this licence, visit http://creativecommons.org/licenses/by/4.0/ The Creative Commons Public Domain Dedication waiver (http://creativecommons.org/publicdomain/zero/1.0/) applies to the data made available in this article, unless otherwise stated in a credit line to the data. 


\section{Background}

Left ventricular assist devices (LVADs) were developed to bridge a patient to heart transplantation [1]. However, as the blood of a patient with an LVAD is exposed to a large surface area of artificial materials, the patient is at a high risk of thrombus formation and thromboembolism. The standard anticoagulation therapy for patients implanted with an LVAD includes warfarin in addition to aspirin therapy [2]. Anticoagulation therapy with warfarin requires precise dose adjustments based on the periodic monitoring of prothrombin time-international normalized ratio (PT-INR) to minimize the risk of bleeding. Many factors, such as drug-drug interactions and lifestyle, can affect a patient's response to warfarin [3, 4]. Sufficient monitoring of these factors is important for the management of warfarin therapy in outpatients. Recently, the development of accurate, portable, point-of-care PT-INR monitors has made possible patient self-testing (PST) as a feasible and convenient anticoagulation management strategy. Furthermore, point-of-care devices, including the CoaguCheck $^{\ominus}$ XS (Roche Diagnostics, Mannheim, Germany) allow PT-INR testing at home [5, 6]. PT-INR PST improves the overall prognosis of patients on lifelong oral anticoagulation therapy because patients remain more precisely within the target therapeutic PT-INR range [7]. However, conventional warfarin therapy in outpatients does not facilitate the sharing of information other than the PT-INR and warfarin dose between patients and care providers in real time.

We developed a cloud-based home medical management information-sharing system named as LVAD@home [8]. The LVAD@home system, an application designed to be used on iPad tablet computers, enables data on multiple factors, such as warfarin dose, selfchecked PT-INR, blood pressure, dietary intake, body condition, body temperature, body weight, urine color, and other physical conditions, to be recorded and shared daily between patients and care providers in real time.
Figure 1 shows the outline using this cloud-based home management system to manage warfarin therapy. However, it has been unclear whether this system is effective in the management of anticoagulation therapy. In this study, we reported cases of outpatients with LVADs using this cloud-based home management system to manage anticoagulation therapy.

\section{Case presentation}

Patient No. 1 was a $40_{\mathrm{S}}$-year-old male with end-stage heart failure owing to a non-ischemic dilated cardiomyopathy underwent an implantation with Heart-Mate $\mathrm{II}^{\circ}$ LVAD (Abbott, IL, USA) and anticoagulation therapy with warfarin was started postoperative Day 1 . The clinical characteristics and outcomes of him are shown in Tables 1 and 2. The target range for PT-INR was set at between 1.8 and 2.6. Before discharge, he attended an education/training program conducted by ward pharmacists for approximately $60 \mathrm{~min}$. In this program, the pharmacist taught important aspects of oral anticoagulation therapy (importance of adherence, target PT-INR ranges, how to use CoaguCheck ${ }^{\bullet} \mathrm{XS}$, signs and symptoms of bleeding and clotting and appropriate actions in the event of these problems, drug interactions, avoiding vitamin K-rich food, and lifestyle issues including dietary intake). If the patient forgot to send data to care providers, the care providers have asked the patients to enter data via LVAD@home system or by telephone. In case of emergency event such as bleeding and pump trouble, the patient must contact hospital by telephone, not LVAD@home system. After discharge (postoperative Day 47), the patient started to use the LVAD@home system to share clinical information with care providers daily. PT-INR was assessed using a CoaguCheck $^{\bullet}$ XS once or twice a week. The pharmacist could evaluate the data based on a treatment algorithm of warfarin developed with physicians [11]. In the case of abnormalities, the pharmacist discussed the data with the physician to

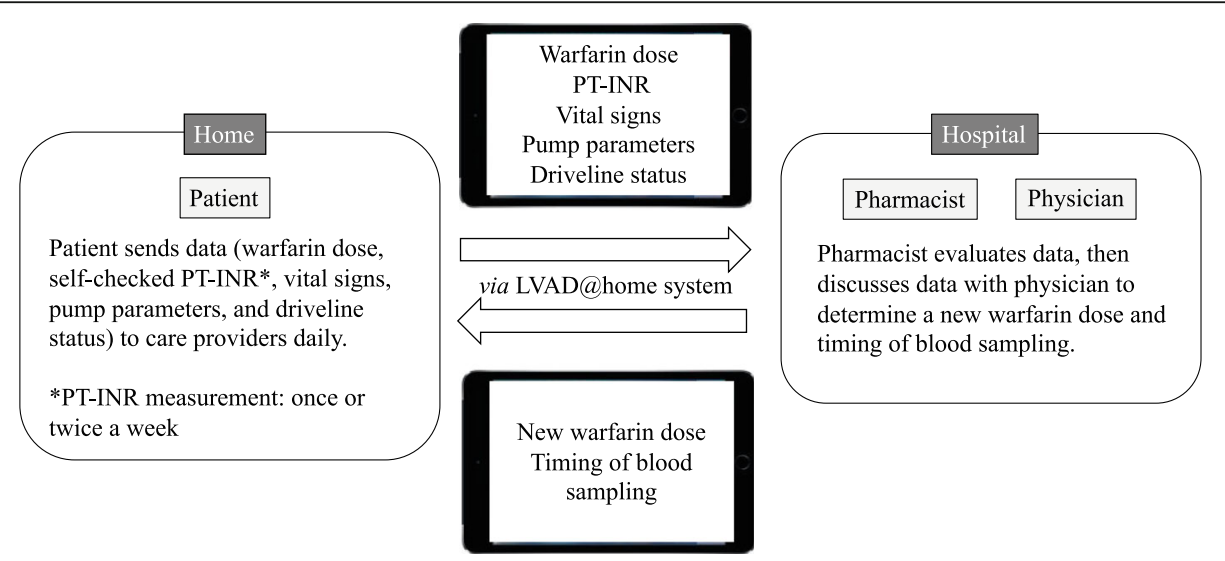

Fig. 1 The outline of warfarin therapy using cloud-based home management system 
Table 1 Baseline clinical characteristics of patients with an LVAD

\begin{tabular}{|c|c|c|c|c|}
\hline No & 1 & 2 & 3 & 4 \\
\hline Gender & Male & Male & Male & Male \\
\hline Age (years) & $40 \mathrm{~s}$ & $10 \mathrm{~s}$ & $50 \mathrm{~s}$ & $40 \mathrm{~s}$ \\
\hline LVAD device & HM II & HM II & HM ॥ & HM II \\
\hline Preimplant device strategy & BTT & BTT & $\mathrm{BTT}$ & BTT \\
\hline Period of LVAD@home system (days) & 581 & 643 & 464 & 400 \\
\hline PT-INR results of CoaguCheck ${ }^{\circledR}$ XS (times) & 53 & 77 & 58 & 51 \\
\hline Co-administration of any antiplatelet agent & Aspirin & Aspirin & Aspirin & Aspirin \\
\hline Target PT-INR & $1.8-2.6$ & $1.8-2.6$ & $1.8-2.6$ & $\begin{array}{l}1.8-2.6 \\
\rightarrow 2.5-3.5\end{array}$ \\
\hline
\end{tabular}

LVAD Left ventricular assist device, PT-INR Prothrombin time-international normalized ratio, HM II Heart-Mate II, BTT Bridge-to-transplant

determine a new warfarin dose and sent the new dose and timing of blood sampling via this system. After hospital discharge, the patient had good control of PT-INR without adverse events, such as bleeding.

On Day 179 after discharge, the pharmacist observed signs of reduced dietary intake on the LVAD@home system. The reason of reduced dietary intake was that the patient was too busy with work. The pharmacist, after consulting the physician, told him to change the timing of the next measurement to earlier than usual (Fig. 2). In addition, the pharmacist explained about the influence of dietary intake on an efficacy of warfarin. On the next day, the patient measured his PT-INR, and found its prolongation from 2.0 to 3.0. Based on this information, the physician decided to decrease the dose of warfarin by $0.5 \mathrm{mg}$. The physician entered the message to reduce the dose of warfarin from $5.0 \mathrm{mg}$ to $4.5 \mathrm{mg}$ via LVAD@home. After taking warfarin, the patient input warfarin dose. The care providers could know that they took appropriate warfarin dose. Thereafter, his dietary intake returned to the baseline and he maintained a stable PTINR value.

Four patients were using this system to monitor warfarin therapy from October 2015 to March 2018. The demographics and clinical characteristics of this study population are shown in Table 1 . The mean age of all patients was $41 \pm 14$ years (mean $\pm \mathrm{SD}$ ) and all were male. All patients underwent LVAD placement as bridge-toheart transplant therapy. They were administered warfarin and aspirin after the LVAD implantation. The target PTINR level of patients No. 1-3 was set between 1.8 and 2.6 by the cardiac surgeon. For patient No. 4 , the goal for PTINR was increased from 1.8-2.6 to $2.5-3.5$ because of the rising lactate dehydrogenase levels. The mean duration of warfarin therapy in all outpatients was $522 \pm 95.3$ days. The mean number of home PT-INR measurements by CoaguCheck $^{\bullet} \mathrm{XS}$ was $59.7 \pm 10.2$.

The clinical outcomes of the four patients are described in Table 2 . The quality of anticoagulation management is expressed as the duration ratio in the target

Table 2 Clinical outcomes of patients with an LVAD

\begin{tabular}{|c|c|c|c|c|c|c|}
\hline No & 1 & 2 & 3 & 4 & Bishop et al. [9] & Ryan et al. [10] \\
\hline TTR (\%) & 88.6 & 92.2 & 89.6 & 90.1 & 44.4 & 74.0 \\
\hline Overall PT-INR, mean \pm SD & $2.01 \pm 0.30$ & $1.87 \pm 0.21$ & $1.92 \pm 0.22$ & $2.47 \pm 0.50$ & $2.32 \pm 0.67$ & - \\
\hline Overall warfarin dose, mean \pm SD & $4.49 \pm 0.76$ & $5.13 \pm 0.58$ & $3.30 \pm 0.34$ & $3.24 \pm 0.33$ & - & - \\
\hline \multicolumn{7}{|l|}{ Major bleeding events } \\
\hline Gl bleeding events & no & no & no & no & yes $(2 / 11)$ & no \\
\hline LVAD site bleeding events & no & no & no & no & no & no \\
\hline Other bleeding events & no & no & no & no & no & no \\
\hline \multicolumn{7}{|l|}{ All thrombosis events } \\
\hline Pump thrombosis events & no & no & no & no & no & no \\
\hline Other thrombosis events & no & no & no & no & yes $^{\mathrm{a}}(1 / 11)$ & yes $^{b}(2 / 162)$ \\
\hline LVAD-related infections & no & yes & yes & no & - & - \\
\hline Heart transplants received & no & yes & no & no & yes $(1 / 11)$ & - \\
\hline
\end{tabular}

$T T R$ Time in therapeutic range, $P T$-INR Prothrombin time-international normalized ratio, Gl Gastrointestinal, LVAD Left ventricular assist devices

${ }^{\mathrm{a} C e n t r a l}$ nervous system thrombosis, ${ }^{\mathrm{b}}$ Deep vein thrombosis 


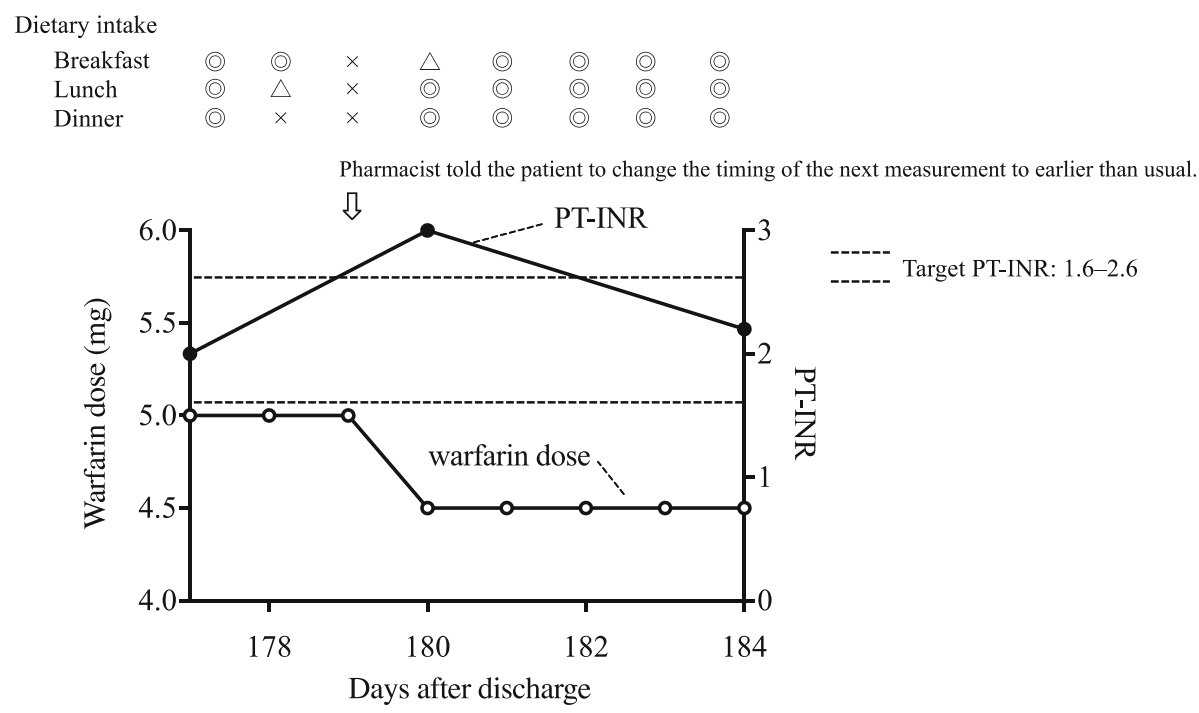

Fig. 2 Time course of warfarin therapy using cloud-based home management system in patient No. 1. Closed and open circles show the PT-INR and warfarin dose, respectively. X-axis represents the days after discharge. Arrows indicate when the pharmacist told the patient to change the timing of the next measurement to earlier than usual

therapeutic range of PT-INR (time in therapeutic range [TTR]) [12]. The mean TTR of all patients was $90.1 \pm$ 1.3. There were no thromboembolic and bleeding events. Furthermore, patient No. 2 underwent a successful heart transplantation after 643 days from starting usage of this system.

\section{Discussion and conclusions}

Telephone and e-mail are usually used to share information on home-based PST, such as warfarin dose and PTINR, between a patient and care providers to manage warfarin therapy $[9,13,14]$. However, with these tools, information on vital signs cannot be shared in real time. A change in vital signs is an important indicator of the anticoagulative efficacy of warfarin; hence, the real-time sharing of this information provides opportunities for early recognition and intervention. Physicians and pharmacists need to access to the health information of a patient in real time to decide on a warfarin dose in clinical practice. Recently, we reported that the anticoagulation activity of warfarin is significantly associated with fasting [15]. When patients experience fasting, more frequent PT-INR measurements and subsequent dose adjustments of warfarin are warranted to avoid adverse effects, such as bleeding. In this study, inadequate anticoagulation (PT-INR was prolonged from 2.0 to 3.0) was observed in one patient due to a decreased dietary intake. Daily follow-up is mandatory, even in the context of home management, and the early detection of reduced dietary intake is important to improve anticoagulation therapy. Using this cloud-based home management system, care providers can remotely track the health information of a patient, such as self-checked PT-INR, warfarin dose, and vital signs, in real time. This system strengthens the communication between the patient and care providers.

The TTR, a surrogate marker for the quality of anticoagulation control, is used to evaluate the clinical effectiveness and safety of warfarin [16]. Significant reduced risk of stroke have been reported in patients with atrial fibrillation taking warfarin whose TTRs are $>70 \%$ compared with those not receiving warfarin [17]. Previous studies have reported that anticoagulation management using PST achieves better control of PT-INR and reduces the risk of bleeding and thrombotic events in patients with atrial fibrillation, venous thromboembolism, and LVADs [9, 10, 18-20]. In this study, using LVAD@home system monitoring, anticoagulation therapy with warfarin was well-controlled in four patients, as their TTR was more than $90 \%$ without thromboembolic and bleeding events. Furthermore, the mean TTR in our study was higher than that in previous studies $[9,10]$. Therefore, the LVAD@home system may improve longterm anticoagulation outcomes in patients implanted with LVAD with PT-INR self-testing at home.

In conclusion, the cloud-based home management system can be applied to share real-time patient information of factors, including dietary intake, that interact with warfarin. It can improve long-term anticoagulation outcomes in patients implanted with LVAD.

\section{Abbreviations}

LVAD: Left ventricular assist devices; PT-INR: Prothrombin time-international normalized ratio; PST: Portable patient self-testing; TTR: Time in therapeutic range 


\section{Acknowledgements}

We thank all staff who were involved in this study.

\section{Authors' contributions}

$Y K, A Y, N K$, and KMatsubara supervised all stages of the project and contributed to the study concept and design, data acquisition, interpretation, and analysis, and critical revision of the manuscript. MU and SN contributed to the study design, research, data analysis, and writing of the manuscript. YS, KMatsumura, and AS contributed to the study design, data acquisition and interpretation, and critical revision of the manuscript. KMinakata, HK, and KMinatoya contributed to study concept and design, data acquisition and interpretation, and critical revision of the manuscript. SI, SN, and TN contributed to study design, data acquisition and interpretation, and critical revision of the manuscript. The author(s) read and approved the final manuscript.

\section{Funding}

This research was partly supported by The Center of innovation Program from MEXT and JST, JSPS Grant-in-Aid for Scientific Research (C) (KAKENHI) (15 K115810001), and a grant from a Kyoto-Funding for innovation in Healthrelated R\&D Fields. None of the authors declare any conflict of interest.

\section{Availability of data and materials}

Data used in this case report will not be shared owing to the risk of identifying an individual.

\section{Ethics approval and consent to participate}

All procedures performed in this study involving human participants were executed in accordance with the ethical standards of the institutional and/or national research committee and with the 1964 Helsinki declaration and its later amendments or comparable ethical standards. This study was approved by the Ethics Committee at Kyoto University Graduate School and Faculty of Medicine (R0174). Written informed consent was obtained from all patients for this case report.

\section{Consent for publication}

A copy of the written consent is available for review by the Editor-in-Chief of this journal.

\section{Competing interests}

The authors declare that they have no competing interest.

\begin{abstract}
Author details
'Department of Clinical Pharmacology and Therapeutics, Kyoto University Hospital, 54 Kawahara-cho, Shogoin, Sakyo-ku, Kyoto 606-8507, Japan. ${ }^{2}$ Department of Health Sciences, Graduate School of Medicine, Osaka University, 2-2 Yamadaoka, Suita 565-0871, Japan. ${ }^{3}$ Department of Cardiovascular Surgery, Graduate School of Medicine, Kyoto University, 54 Kawahara-cho, Shogoin, Sakyo-ku, Kyoto 606-8507, Japan. ${ }^{4}$ Department of Human Health Science, Graduate School of Medicine, Kyoto University, 53 Kawahara-cho, Shogoin, Sakyo-ku, Kyoto 606-8507, Japan.
\end{abstract}

Received: 19 November 2020 Accepted: 29 December 2020 Published online: 01 February 2021

\section{References}

1. Kirklin JK, Naftel DC, Kormos RL, Stevenson LW, Pagani FD, Miller MA, et al. Fifth INTERMACS annual report: risk factor analysis from more than 6,000 mechanical circulatory support patients. J Heart Lung Transplant. 2013;32: 141-56.

2. Feldman D, Pamboukian SV, Teuteberg JJ, Birks E, Lietz K, Moore SA, et al. The 2013 International Society for Heart and Lung Transplantation guidelines for mechanical circulatory support: executive summary. J Heart Lung Transplant. 2013;32:157-87.

3. Yamamoto H, Habu Y, Yano I, Ozaki J, Kimura Y, Sato E, et al. Comparison of the effects of azole antifungal agents on the anticoagulant activity of warfarin. Biol Pharm Bull. 2014;37:1990-3.

4. Walter-Sack I, Klotz U. Influence of diet and nutritional status on drug metabolism. Clin Pharmacokinet. 1996;31:47-64.
5. Plesch W, van den Besselaar AM. Validation of the international normalized ratio (INR) in a new point-of-care system designed for home monitoring of oral anticoagulation therapy. Int J Lab Hematol. 2009;31:20-5.

6. Torreiro EG, Fernández EG, Rodríguez RM, López CV, Núñez JB. Comparative study of accuracy and clinical agreement of the CoaguChek XS portable device versus standard laboratory practice in unexperienced patients. Thromb Haemost. 2009;101:969-74.

7. Horstkotte D, Piper C, Wiemer M. Optimal frequency of patient monitoring and intensity of oral anticoagulation therapy in valvular heart disease. J Thromb Thrombolysis. 1998;1:19-24.

8. Nomoto S, Utsumi M, Minakata K. A cloud-based home management system for patients with a left ventricular assist device: a case report. Int J Artif Organs. 2016:39:245-8.

9. Bishop MA, Streiff MB, Ensor CR, Tedford RJ, Russell SD, Ross PA. Pharmacistmanaged international normalized ratio patient self-testing is associated with increased time in therapeutic range in patients with left ventricular assist devices at an academic medical center. ASAIO J. 2014;60:193-8.

10. Ryan F, Byrne S, O'Shea S. Randomized controlled trial of supervised patient self-testing of warfarin therapy using an internet-based expert system. J Thromb Haemost. 2009:7:1284-90.

11. Katada Y, Nakagawa S, Minakata K, Odaka M, Taue H, Sato $Y$, et al. Efficacy of protocol-based pharmacotherapy management on anticoagulation with warfarin for patients with cardiovascular surgery. J Clin Pharm Ther. 2017;42: 591-7.

12. Rosendaal FR, Cannegieter SC, van der Meer FJ, Briët E. A method to determine the optimal intensity of oral anticoagulant therapy. Thromb Haemost. 1993;69:236-9.

13. Yamamura K, Yano K, Hirooka Y, Hirashiki A, Murohara T, Yamada K. A successful case of a patient undergoing warfarin and S-1 therapy using internet-based control of home-measured PT-INR. Yakugaku Zasshi. 2015; 135:925-7.

14. Mantha S, Pianka AM, Tsapatsaris N. Determinants of intracranial hemorrhage incidence in patients on oral anticoagulation followed at the Lahey clinic. J Thromb Thrombolysis. 2011;32:334-42.

15. Katada Y, Nakagawa S, Nishimura A, Sato YK, Taue H, Matsumura K, et al. Effects of fasting on warfarin sensitivity index in patients undergoing cardiovascular surgery. Eur J Clin Pharmacol. 2019:75:561-8.

16. Rose AJ, Hylek EM, Ozonoff A, Ash AS, Reisman Jl, Berlowitz DR. Riskadjusted percent time in therapeutic range as a quality indicator for outpatient oral anticoagulation: results of the veterans affairs study to improve anticoagulation (VARIA). Circ Cardiovasc Qual Outcomes. 2011;4: $22-9$

17. Morgan CL, McEwan P, Tukiendorf A, Robinson PA, Clemens A, Plumb JM. Warfarin treatment in patients with atrial fibrillation: observing outcomes associated with varying levels of INR control. Thromb Res. 2009;124:37-41.

18. Heneghan C, Ward A, Perera R. Self-monitoring Trialist collaboration; Bankhead C, fuller a, et al. self-monitoring of oral anticoagulation: systematic review and meta-analysis of individual patient data. Lancet. 2012:379:322_ 34

19. Bloomfield HE, Krause A, Greer N, Taylor BC, MacDonald R, Rutks I, et al. Meta-analysis: effect of patient self-testing and self-management of longterm anticoagulation on major clinical outcomes. Ann Intern Med. 2011;154: 472-82.

20. Matchar DB, Jacobson A, Dolor R, Edson R, Uyeda L, Phibbs CS, et al. Effect of home testing of international normalized ratio on clinical events. $\mathrm{N}$ Engl J Med. 2010;363:1608-20.

\section{Publisher's Note}

Springer Nature remains neutral with regard to jurisdictional claims in published maps and institutional affiliations. 